\title{
Luminescence and photoconductivity of poly-n-epoxypropylcarbazole with intramolecular charge transfer compounds
}

\author{
N.A.Davidenko, N.G.Kuvshinsky, V.G.Syromiatnikov \\ Kiev Taras Shevchenko University, 64, vul. Volodymirs'ka, Kyiv, 01033, Ukraine
}

\begin{abstract}
Features of charges photogeneration in the films of poly-N-epoxypropylcarbazole doped with compounds with intramolecular charge transfer used in holographic recording media are investigated. Influence of an external electric field on the quantum yield of charges photogeneration and photoluminescence as studied. Different dependencies on the temperature of such two parameters within the wide temperature range were shown. The assumption of the tunnel mechanism of electron-hole pair formation from an excited molecule of compound with intramolecular charge transfer was made.
\end{abstract}

Keywords: amorphous molecular semiconductor, electron-hole pair, photoluminescence, photoconductivity.

Paper received: 08.10.99; revised manuscript received: 23.12.99; accepted for publication 04.01.00.

\section{Introduction}

The present researches deal with a photoconductivity of amorphous molecular semiconductor films based on polyepoxypropylcarbazole (PEPC) containing compounds with intramolecular charge transfer (CICT). Features of combined electron-hole pairs (EHP) formation, annihilation and dissociation are studied.

The basic outcomes were obtained during the investigations of an electric conductivity, photoconductivity as well as recombination luminescence and photoluminescence in dependency on the CICT structure, temperature and the strength of applied electric and magnetic fields.

It was shown that the efficiency of charge carriers photogeneration is proportional to a difference between the ionization potential of the CICT molecules and PEPC carbazole nuclei. An increase of this difference correlates with growth of an initial distance between charges in EHP and of a positive potential barrier for the hole coming back into the recombination center after EHP dissociation. Both these factors involve a growth of EHP dissociation probability. It should be pointed out, that the EHP lifetime can reach tens hours at room temperature, but in the course of EHP relaxation, spatial distribution by distances between charges takes place. It was concluded in $[2,3]$, that during photogeneration as well as thermogeneration the hole tunnels from the high valence electronic level of the excited CICT molecule into the valence electronic level of the carbazole nuclei which take part in a creation of the energy band for the holes transport. The probability of EHP dissociation increases with creation of an additional transport energy band when introducing acceptor molecules into the investigated films with affinity energy to electrons not less, than affinity energy of the CICT molecule with respect to the electron [1]. It is these films that are used as high sensitive holographic recording media.

We have investigated the effects of external electric field influence on photoconductivity and photoluminescence of PEPC films with CICT to confirm the assumetion of tunnel mechanism of EHP formation and annihilation. This technique is enough informative [4]. It allows to study the first stage of photogeneration, i.e. formation of EHP.

\section{Samples and experimental methods}

The object for investigation were the samples of sandwich structures Al-AMS-SnO 2 . The samples were prepared by casting of solution in toluene onto the glass substrates coated by the thin $\mathrm{SnO}_{2}$ film. After drying then in a vacuum chamber, the thickness of AMS films were 1-2 $\mu \mathrm{m}$. Then aluminum electrodes were deposited onto the substrates under the pressure $10^{-5}$ Torr in a vacuum chamber.

For investigation the next AMS were used: PEPC $+N_{1}$ mol. $\%$ GD-TENF $+N_{3}$ mol. $\%$ Ud-DDFK, PS $+N_{1}$ mol. $\%$ GD-TENF $+N_{2}$ mol. $\%$ IPC $+N_{3}$ mol. $\%$ UdDDFK, where GD-TENF is 9-(4-heptadecyl-1,3-dithiol- 


\section{N.A. Davidenko et al.: Luminescence and photoconductivity of poly-n-...}

2-iliden)-2,4,5,7-tetranitrofluorene (CICT), IPC is N-isopropyl-carbazole (donor molecules), Ud-DDFK is 2,7dinitro-9-dicyan-methylene/fluorene-4-oic acid (acceptor molecules). PS is polystyrole. Concentrations of GDTENF $N_{1}$, IPC $N_{2}$ and Ud-DDFK $N_{3}$ molecules were varied in the ranges of $1-5,0-20,0-20$ mol.\% respectively.

The influence of electric field on PL was investigated in monochromatically illuminated samples of sandwich structures using an electric voltage on the $\mathrm{Al}$ and $\mathrm{SnO}_{2}$ electrodes. Light beam was directed at the angle of $85^{\circ}$ from the side of $\mathrm{SnO}_{2}$ electrode transparent in the visible and near IR parts of spectrum. PL emission was registered from the side of the same $\mathrm{SnO}_{2}$ electrode normally to the sample surface. The samples was illuminated by the laser with the wavelength of irradiation $\lambda=633 \mathrm{~nm}$, which corresponds to the absorption maximum (spectral dependencies of the absorption coefficient $(K)$ (curve 1), and intensity of PL I (curve 2) for the films PEPC + 2mol. $\%$ GDTENF are shown in Fig. 1). Intensity of illumination was changed by the light filters from 5 to $500 \mathrm{~W} / \mathrm{m}^{2}$. Accuracy of $I$ measurements was not worse than $5 \cdot 10^{-4}$.

Investigation of PL $I$ at different temperatures was carried out by placing of samples in the vacuum cryostat with optical windows. Thermostatic accuracy was $\pm 0.1 \mathrm{~K}$. For the registration of PL $I$, instead of the monochromator, a wide-band light filter was used. Possible influence of reflected laser beam was neutralized by subtraction of PL signals and light scattered on the mirror glass without the AMS film, which was placed instead of the sample investigated.

We have ascertained that electric field causes the decrease of PL $I$ in the samples with $N_{1}=1-5, N_{2}=0.5-20$ and $N_{3}=0.5-20 \mathrm{~mol} . \%$ and does not influence on $I$ in the samples Al-PS $+N_{1}$ mol. $\%$ GD-TENF-SnO 2 .

\section{Results and discussion}

The curve 1 in Fig. 2 represents the kinetics of PL $I(t)$ in the samples A-PS $+2 \mathrm{~mol} . \% \mathrm{GD}-\mathrm{TENF}+1 \mathrm{~mol} . \% \mathrm{Ud}-$ DDFK-SnO ${ }_{2} . t_{1}$ and $t_{5}$ are the moments of illumination

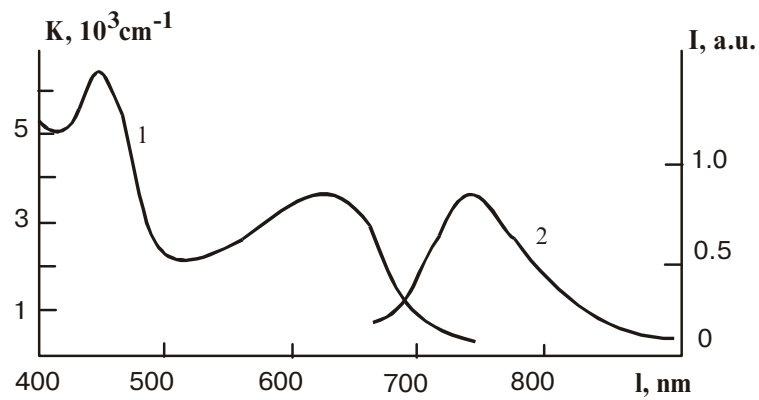

Fig. 1. Spectra of absorbtion (curve 1) and photoluminescence (curve 2) for the film PEPC $+2 \mathrm{~m} . \%$ GD-TENF under the temperature $T=293 \mathrm{~K}$. beginning, $t_{2}$ and $t_{3}$ are the moments of electric voltage switching on and switching off, respectively, $t_{4}$ is the moment of the illumination stopping. Curve 2 of this figure corresponds to the dependence $I(t)$ without influence of electric field.

The decrease of PL $I$ was observed in all investigated samples. This effect is reversible, but the velocity of $I$ restoration in darkness increases with growth of $N_{2}$ or $N_{3}$ concentrations. After turning on the electric voltage (moment $t_{2}$ in Fig. 2), magnitude of $I$ sharply (for the time interval less than the time constant of setup $0.05 \mathrm{~s}$ ) decreases by the value $D I$ and further the velocity of $I$ decrease differs from that observed without electric voltage (curves 1 and 2 in Fig. 2). After switching off the electric voltage (moment $t_{3}$ on Fig. 2), I magnitude abruptly increases and then slowly decreases with the same velocity as without electric voltage.

Thus, switching on the electric voltage is accompanied by two effects: quick and slow PL I reduction. These effects may be parted in time and may be studied each taken separately.

Respective value $\Delta I / I$ of quick component of $I$ decay kinetics not depends neither on time after the beginning of illumination up to the moment of voltage switching on, nor on the light wavelength and light intensity. This value is equal to the $\Delta I / I$ of quick component of restoration kinetics after the voltage switching off (the time moments $t_{2}$ and $t_{3}$, respectively, in Fig 2).

In Fig. 3 the dependencies $\Delta I / I$ on $N_{2}$ and $N_{3}$ in the samples Al-PS +2 mol. $\%$ GD-TENF $+\mathrm{N}_{2}$ mol. $\%$ IPC$\mathrm{SnO}_{2}$, (curvel) and Al-PS + 2m. $\%$ GD-TENF+N 3 m. $\%$ UdDDFK- $\mathrm{SnO}_{2}$ (cur-ve 2) are plotted. In the same figure doted horizontal lines indicate the level of $\Delta I / I$ in the samples Al-PEPC + 2mol. $\%$ GD-TENF-SnO 2 , (curve 3 ) and $\mathrm{Al}-\mathrm{PEPC}+2 \mathrm{~mol} \% \mathrm{GD}-\mathrm{TENF}+5 \mathrm{~mol} \% \mathrm{Ud}-\mathrm{DDFK}-$ $\mathrm{SnO}_{2}$, (curve 4). Dependencies $\Delta I / I\left(N_{2}\right)$ and $\Delta I / I\left(N_{3}\right)$ reach the saturation for $N_{2}, N_{3} \sim 10 \mathrm{~mol} . \%$, but the magnitude of $\Delta I / I$ would be much greater if the AMS film contains of the donor and acceptor molecules. For example if PEPC

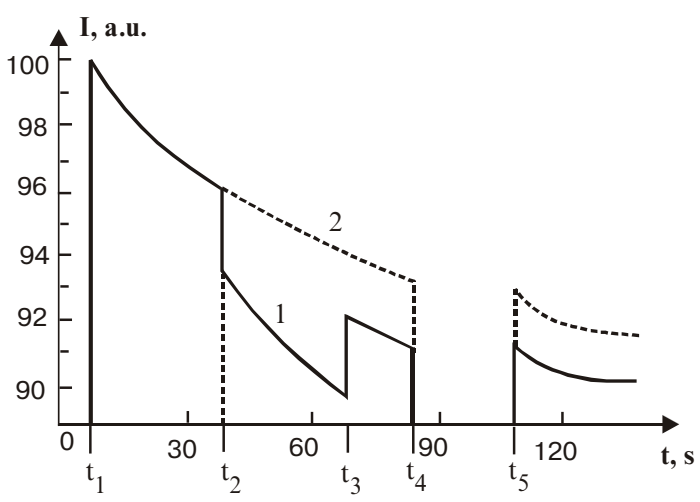

Fig. 2. Kinetics of photoluminescence intensity during the illumination of the sample Al-PS $+2 \mathrm{~m} . \% \mathrm{GD}-\mathrm{TENF}+1 \mathrm{~m} . \% \mathrm{Ud}-\mathrm{DDFK}$ $\mathrm{SnO}_{2}$, under the applied electric voltage $\left(\mathrm{E}=1.1 \cdot 10^{8} \mathrm{~V} / \mathrm{m}\right)($ curve 1 ) and without electric voltage (curve 2). 


\section{N.A. Davidenko et al.: Luminescence and photoconductivity of poly-n-...}

with GD-TENF consists of Ud-DDFK, then instead of curve 3 in Fig.3, curve 4 would be, valid.

In Fig. 4 curve 1 represents the dependence $\Delta I / I(E)$ in the coordinates $\lg \Delta I / I$ and $E^{1 / 2}$. In the same figure curve 2 corresponds to the dependence of quantum yield of current carriers photogeneration $(\eta)$ on $E$ in the coordinates $\operatorname{lgh}$ and $E^{1 / 2}$. This curve was achieved using dependence $\eta(E)$ in [2]. Within the range of electric field strengths $9 \cdot 10^{7}<E<2,5 \cdot 10^{8} \mathrm{~V} / \mathrm{m}$ the dependence $\lg \Delta I / I\left(E^{1 / 2}\right)$ is not linear and absolute values $\Delta I / I$ is less than $\eta$. This fact

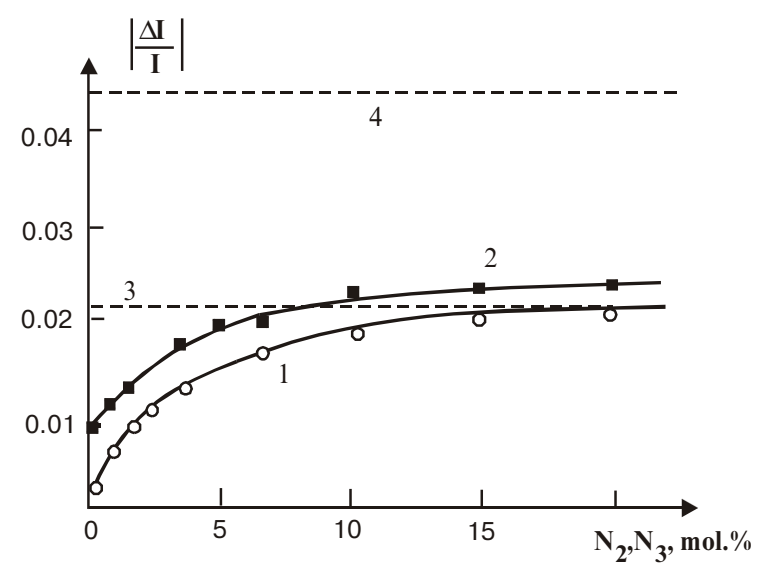

Fig. 3. Dependence $|\Delta I / I|$ on concentration $N_{2}$ of IPC molecules (curve 1) and on concentration $N_{3}$ of Ud-DUFK molecules (curve 2) in the samples Al-PS $+2 \mathrm{~m} . \%$ GD-TENF $+\mathrm{N}_{2} \mathrm{~m} . \%$ IPC-SnO 2 or Al-PS $+2 \mathrm{~m} . \%$ GD-TENF $+N_{3} \mathrm{~m} . \%$ Ud-DDFK-SnO 2 . Curve 3 and 4 represents the level of $|\Delta \mathrm{I} / \mathrm{I}|$ in the sample Al-PEPC + $2 \mathrm{~m} \% \mathrm{GD}-\mathrm{TENF}-\mathrm{SnO}_{2}$, and Al-PEPC + 2m.\%GD-TENF + $5 \mathrm{~m} . \% \mathrm{Ud}-\mathrm{DDFK}-\mathrm{SnO}_{2}$ respectively $E=1 \cdot 10^{8} \mathrm{~V} / \mathrm{m}, T=293 \mathrm{~K}$.

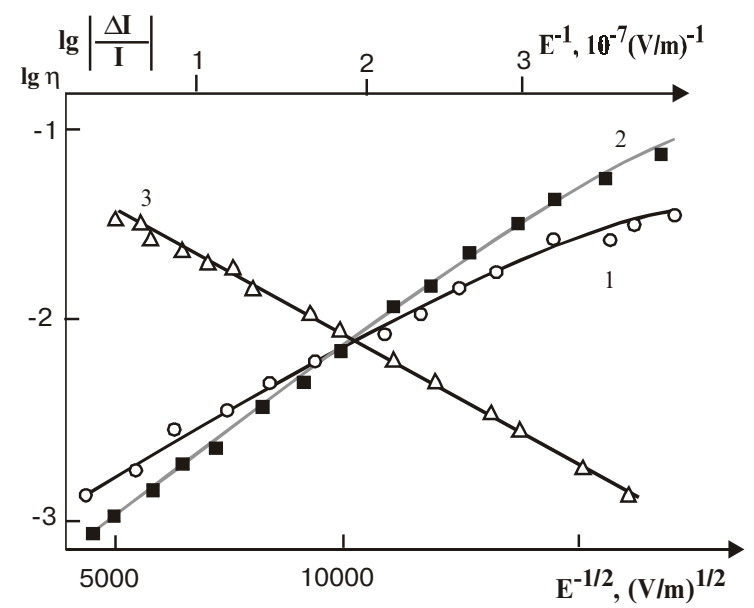

Fig. 4. Dependencies $\lg \Delta \mathrm{I} / \mathrm{I}\left(E^{1 / 2}\right)$ (curve 1), $\lg \eta\left(E^{1 / 2}\right)$ (curve 2$), \lg \Delta \mathrm{I} / \mathrm{I}\left(\mathrm{E}^{-1}\right)$ (curve 3 ) in the sample Al-PEPC $+2 \mathrm{~m} . \%$ GD-TENF-SnO ${ }_{2}$ under $T=293 \mathrm{~K}$ means that dependence $\Delta I / I(E)$ does not coincides with analogous dependence of quantum yield of photo-generation. Curve 3 in Fig. 4 represents the dependence of $\Delta I / I$ on $E$ in the coordinates $\lg \Delta I / I$ and $E^{-1}$. This dependence may be linearly approximated.

There are the dependencies $\lg \Delta I / I(1 / T)$ for five $E$ values in Fig.5. Within the temperature interval $77<T<$ $230 \mathrm{~K}$ the magnitude $\Delta I / I$ is independent of $T$. Within the temperatures interval $240<T<340 \mathrm{~K}$ the dependence $\lg \Delta I / I(T)$ has an activation character. Activation energy of $\Delta I / I$ does not depend on $E$ and is equal $W_{a}=0.086 \pm$ $0.005 \mathrm{eV}$.

Slow component of PL decae kinetics in electric fields depends on $E, N_{2}$, and $N_{3}$. The velocity of $I$ reduction increases with $E$. Increase of $N_{2}$ and $N_{3}$ is accompanied by the decrease of slow component of kinetics. This component is actually undetectable in the samples Al-PEPC + $2 \mathrm{~mol} . \% \mathrm{GD}-\mathrm{TENF}+5 \mathrm{~mol} . \% \mathrm{Ud}-\mathrm{DDFK}-\mathrm{SnO}_{2}$.

Now we shall name some possible causes of PL I decrease in electric field.

1. Growth of probability of EHP dissociation and consequently decrease of both probability of EHP annihilation and probability of recombination luminescence [5].

2 . Increase of free charge carriers concentration which influences on the processes of intramolecular conversion of light absorption centers (CICT) and on the change of correlation between the probabilities of radiative and radiationless transitions of these centers in favor of the latter one [6].

3. In electric field the transparency of potential barrier for the electron tunneling transition between the molecules increases, and the time of this transition decreases. As a result, for the CICT molecules occupied, the ground excited singlet state increase of autoionization probability takes place.

The first reason among the mentioned above is hardly probable because, for temperature more than $280 \mathrm{~K}$, recombination luminescence has practically zero quantum

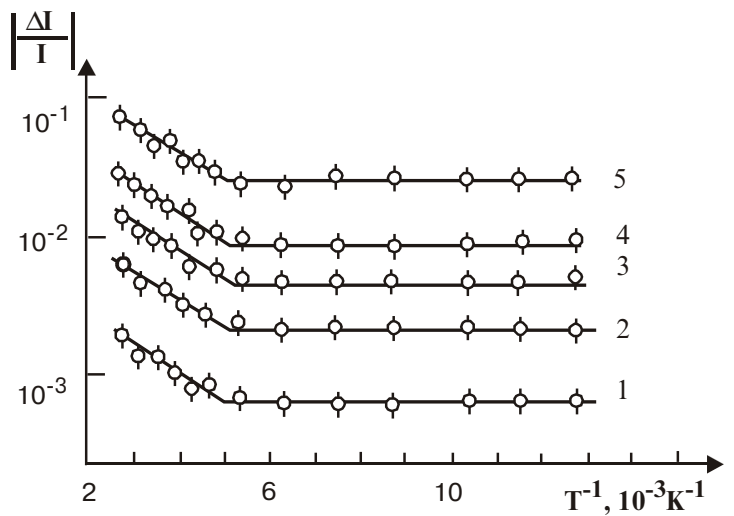

Fig. 5. Dependencies $\lg \Delta \mathrm{I} / \mathrm{I}(1 / \mathrm{T})$ in the sample Al-PEPC $+2 \mathrm{~m} . \%$ GD-TENF-SnO ${ }_{2}$, lor the electric field strengths $\mathrm{E}=5 \cdot 10^{7} \mathrm{~V} / \mathrm{m}(1)$, $8 \cdot 10^{7}(2), 1 \cdot 10^{8}(3), 1.1 \cdot 10^{8}(4), 1.5 \cdot 10^{8}(5)$. 


\section{N.A. Davidenko et al.: Luminescence and photoconductivity of poly-n-...}

yield [7]. Recombination luminescence may be identified [8] in accordance to specific dependence $I(t) \sim t^{-m}$ after the illumination switching off. Moreover, if the following correlation between $\Delta I / I$ and $h$ is true

$\eta+|\Delta I / I|=1$

as it was supposed in [7], then the dependencies $1-|\Delta I / I|(E, T)$ and $\eta(E, \mathrm{~T})$ would coincide. But, as it follows from Fig. 4 and 5 these dependencies do not coincide. The dependencies $\Delta I / I\left(N_{2}\right)$ and $\eta\left(N_{2}\right)$ in the samples Al-PS + $N_{1}$ mol.\%GD-TENF $+\mathrm{N}_{2}$ mol. $\%$ IPC-SnO 2 and dependencies $\Delta I / I\left(N_{3}\right)$ and $h\left(N_{3}\right)$ in the samples Al-PS + $\mathrm{N}_{1}$ mol.\%GD-TENF $+\mathrm{N}_{3}$ mol.\% Ud-DDFK-SnO 2 (see Fig. 3) do not correlate, too (for example in the samples $\mathrm{Al}-\mathrm{PS}+2 \mathrm{~mol} . \% \mathrm{GD}-\mathrm{TENF}+10 \mathrm{~mol} . \% \mathrm{IPC}-\mathrm{SnO}_{2}$ for $E=$ $1 \cdot 10^{8} \mathrm{~V} / \mathrm{m} \Delta I / I=0.02$, but $\left.\eta=0.001\right)$.

The second reason afore mentioned of the quick $E$ influence on $I$ does not fully explain experimental facts, too. The concentrations of free current carriers $(n)$ changed in electric field as the result of alteration both of EHP dissociation probability and electrons and holes mobilities $(\mu)$. Dependencies of $\eta$ and $\mu$ on $E$ and $T$ has a exponential character. Dependence $n(E, T)$ may be represented in accordance to [1]. Therefore, it would be natural to assume that under the independence of ions effective radius for PL decay from $n$, dependencies of $\Delta I / I$ on $E$ and $T$ must consists of exponential component too and activation energies of $\Delta \mathrm{I} / \mathrm{I}$ would depend on $E$. But experimental results represented in Fig. 4 and 5 do not confirm this assumption and demonstrate more complicate character of $\Delta I / I(E, T)$ dependence. It must be noted that without electric field in the films PS $+N_{1}$ mol.\%GD-TENF + 20mol.\%IPC where longliving EHP arise with un-correlated charge spins [2] and in the films PS $+N_{1}$ mol. $\%$ GDTENF where such EHP do not arise the values of $I$ are the same for identical film thicknesses. Thus, the quick component of PL decay kinetics is not determined by the increase of paramagnetic particles number in AMS films.

The third from the mentioned reasons of $E$ influence on $I$ is the most appropriate for the explanation of the experimental datas plotted in Figs. 3 to 5. Independence of $\Delta I / I$ from $T$ within the temperatures interval $77<T<$ $230 \mathrm{~K}$ means that the quick PL decay is probably connected with the intramolecular electron transitions (in the films PS $+N_{1}$ mol. $\%$ GD-TENF EHP do not arise, and the influence of $E$ on $I$ is absent). These transitions are accomplished without activation due to tunneling transitions of either hole from CICT to IPC or electron from CICT to Ud-DDFK. For temperatures $T>240$ the probability of such transitions increases due to the additional activation mechanism. Influence of electric field may be considered as the increase of transparency of a potential barrier between the CICT and IPC or between the CICT and Ud-DDFK. In Fig. 4 the dependence $\Delta I / I(E)$ in coordinates $\lg \Delta I / I$ and $E^{-1}$ for the sample Al-PEPC + 2 mol.\%GD-TENF-SnO 2 is plotted (curve 3). Analogous dependencies were obtained for all investigated samples. Linear character of all dependencies is on evidence of change of barrier transparency in electric field [9].
It may be concluded from the increase of intermolecular transitions probability and decrease of probability of intramolecular emission transitions under the electric field growth that excited state of CICT molecule (from which the radiative transition in the ground state of CICT occurs) is a singlet state. The latter means that in strong electric fields quantum yield of singlet EHP generation increases. Part of additional singlet EHP concentrations in $\eta$ will be appreciable only for $E>1 \cdot 10^{8} \mathrm{~V} / \mathrm{m}$ because, as it was shown in [10], in AMS with CICT only triplet EHP arise which then dissociate, and free current carriers appear. We have observed the increase of photogeneralion efficiency of current carriers in external magnetic field. This experimental fact confirms the assumption about preferable photogeneration of triplet EHP in AMS with GD-TENF during the first stage of photoge-neration. This effect is independent from the orientation of the samples with sandwich structures relatively to the magnetic field direction and reaches a saturated state in magnetic fields $H<1 \mathrm{KOe}$. In accordance to [11] it is evident that both change of EHP multiplicity during its dissociation accordingly to mechanism of ultra-fine interaction and of predominance of triplet EHP (with great lifetime) concentration during the first stage of photogeneration in comparison with singlet EHP (with smaller lifetime) concentration take place. That is why, part of additional singlet EHP in efficiency of current carriers photogeneration may be considerable when the time of EHP dissociation decreases and becomes comparable with lifetime of singlet EHP that is under the sufficiently high electric field strength. This effect was apparently observed in [2].

Respectively to quick kinetics component of influence of $E$ on $I$ the following must be noted. Increase of $N_{2}$ and $N_{3}$ concentrations in AMS films involves the growth of $\Delta I / I$ values, because the velocity of intramolecular transitions increases when the average distance between the molecules decreases. This effect was studied experimentally, and it is reflected in Fig. 3 by curves 1 and 2. However, these intramolecular transitions would become more probable if the opportunities of exit from CICT both hole and electron exist. This probability growth is corroborated by the sufficient increase of DI/I under the presence of acceptor molecules in the film PEPC $+\mathrm{N}_{1}$ mol.\%GDTENF (see curves 1, 3 and 4 in Fig. 3).

We have considered the possible causes of appearance of slow component in PL decay observed in electric field. Three principal by possible reasons exist: field;

1. Acceleration of photochemical reactions in electric

2. Growth of free current carriers concentrations during the EHP dissociation in films with small mobility of carriers which accumulate in the volume of film, turn into the paramagnetic particle and has a great effective radius of PL extinguishing;

3. Increase of long-lived EHP concentration in triplet state which has spin 1 and involve the PL extinguishing too [6].

Among the listed causes, the first one has a small probability because we have attempted to register the 


\section{N.A. Davidenko et al.: Luminescence and photoconductivity of poly-n-...}

absorbtion of photochemical reactions products, but we haven't do it.

It is known [6], that the paramagnetic particles with spin 1/2 (free charge carriers) and with spin 1 (EHP with correlated spins) has a great radius of PL extinguishing. Concentration of such paramagnetic centers decreases under the growth of $\mathrm{N}_{2}$ or $\mathrm{N}_{3}$, because increase of holes or electrons mobilities in AMS take place. Consequently life time of these paramagnetic centers falis. However rise of velocity of PL I extinguishing under the increase of $E$ determined principally by the second from the mentioned reasons, because under these circumstances the probability of EHP dissociation increases but concentration of long-lived EHP decreases. Besides that, during the investigations of illuminated AMS films by ESR method we haven't found the para-magnetic particles with integer spins.

On the base of experimentally achieved results the following scheme of the degradation of electron excitation energy of CICT in AMS may be proposed:

Supposed scheme is represented in Fig. 6 where radiationless electron transitions between the CICT molecules and tunneling electron transitions between CICT and acceptor molecule (A) are plotted by the broken lines and intramolecular transitions under the absorbtion and emission of light quantum are plotted by the continuous lines. As it is shown in scheme 3 in Fig. 6 after the light quantum $h v$ absorbtion CICT molecule from the ground singlet state ${ }^{1} \mathrm{C}_{0}$ passes into the one among the excited singlet states ${ }^{1} \mathrm{C}_{2}$. As result of intramolecular spin conversion and radiationless degradation of the some part of electron excitation energy the CICT molecule from the state ${ }^{1} C_{2}$ passes either into the lower singlet excited state ${ }^{1} \mathrm{C}_{1}$ or into the lower triplet excited state ${ }^{3} \mathrm{C}_{1}$. As the result of charges tunneling from CICT in the state ${ }^{3} \mathrm{C}_{1}$ with velocity constant $k_{3}$ in AMS consists of both donor and acceptor molecules EHP in triplet state ${ }^{3}\left(p^{+} e^{-}\right)$appears. Singlet $\mathrm{EHP}^{1}\left(p^{+} e^{-}\right)$appears either from ${ }^{3}\left(p^{+} e^{-}\right)$accordingly to mechanism of ultra-fine interaction with velocity con-

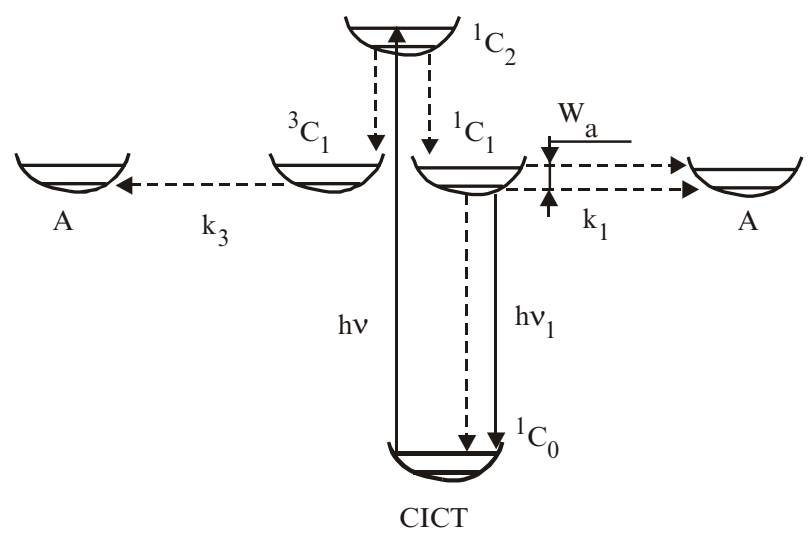

Fig. 6. Scheme of energy degradation of CICT electron excitation. stant $k_{\mathrm{ST}}$ or due to the CICT molecules autoionization from the state ${ }^{1} \mathrm{C}_{1}$ with velocity constant $k_{1}$. Velocity constant $k_{1}$ and consequently the probability of singlet EHP generation rise under the increase of E. Emission (with light quantum $h v_{1}$ emission) or radiationless transition into the ground state accomplishes from the singlet state ${ }^{1} \mathrm{C}_{1}$. Singlet EHP annihilate with velocity constant $k_{a}$. Generation of free current carriers $p^{+} \ldots e^{-}$occurs during the singlet and triplet EHP dissociation with velocity constant $k_{\eta}$ Intermolecular electron transitions between CICT and acceptor molecule $\mathrm{A}$ (or donor molecule) may realize both from the lower vibration electron level of the state ${ }^{1} \mathrm{C}_{1}$ and from the more high vibration level which has energy greater by value $W_{a}=0.1 \mathrm{eV}$.

\section{Conclusions}

1. Within the temperatures interval $280<\mathrm{T}<340 \mathrm{~K}$ photolummescence of AMS is not recombination. It accomplishes due to the emission energy degradation of excited CICT molecules in a singlet state.

2. In an external electric field the potential barrier transparency increases for tunneling transition of holes and electrons to the neighbor donor and acceptor molecules, respectively. This is the cause of quick PL extinguishing and singlet EHP generation.

3. In AMS with small charge carriers mobilities the life time of carriers is greater than the time of spin-lattice relaxation. This is the cause both of paramagnetic particles accumulation on the volume of AMS film and of the PL extinguishing by the paramagnetic particles in an external electric field.

\section{References}

1. N.G Kuvshinsky, N.A. Davidenko, V.M. Komko. Physics of Amorphous Molecular Semiconductors. Kiev, Lybid', 1994.

2. N.A. Davidenko, N.G. Kuvshinsky. Peculiarities of generation and dissociation of electron-hole pairs in amorphous molecular semiconductors containing molecules of compounds with intramolecular charge transfer. // J. Inf. Rec. Mats., 21, pp.185-197 (1993).

3. N.A. Davidenko, N.G. Kuvshinsky. Electrical conductivity of amorphous molecular semiconductors containing compounds with intramolecular charge transfer. // J.Inf.Recording, 24, pp.289-294 (1996).

4. N.A. Davidenko, A.A. Ishchenko. Spin conversion and electric field effect on photoluminescence in amorphous molecular semiconductors doped with ionic dyes. // Chem. Phys., 247, pp.237-243 (1999).

5. J. Kalinowski. Electron processes in organic electroluminescence. In R.W.Munn, A.Miniewicz, B.Kuchta; Electrical and Related Properties of Organic Solids. Kluwer Academic Publishers, Printed in the Netherlands, pp.167-206 (1997).

6. A.N. Terenin, Photophysics of dye molecules and related compounds, Leningrad, Nauka (1967).

7. N. Davidenko, A. Ischenko, A. Kadaschuk, N. Kuvshinsky, N. Ostapenko, Yu. Skryshevsky. Study of photoconductivity and luminescence of amorphous molecular semiconductors doped by polymethine dyes. // Functional Materials, 5(3), pp.353-355 (1998). 
N.A. Davidenko et al.: Luminescence and photoconductivity of poly-n-...

8. N.A. Davidenko, A.K. Kadashchuk, N.G. Kuvshinsky, N.I. Ostapenko, N.V. Lukashenko. Photoconductivity features of amorphous molecular semiconductor films containing compounds with intramolecular charge transfer. The natue of capture centers // J.Inf.Recording, 24, pp.327-338 (1996).

9. L.D. Landau, E.M. Lifshits, Quantum Mechanics. Vol. 3 Nonrelativistic Theory, Nauka, Moscow (1989), (in Russian).
10. N.A. Davidenko, N.G. Kuvshinsky, Multiplicity of electronhole pairs and its change while in photogeneration of current carriers in amorphous molecular semiconductors. // J. Inf. Rec. Mats., v.21, pp.37-45, (1994).

11. K.M. Salikhov, Yu. N. Molin, R.Z. Sagdeev, A.L. Buchachenko, Spin Polarization and Magnetic Effects in Radical Reactions, Akademial Kiado, Budapest (1984). 